\title{
Sensibilidade ao Contraste de Crianças Surdas e Ouvintes para Grades Senoidais em Condições Mesópicas
}

\author{
Contrast Sensitivity of Deaf and Hearing Children for Sine-Wave Gratings \\ in Mesopic Conditions
}

\author{
Natanael Antonio dos Santos*, Liana Chaves Mendes \& \\ Príscilla Anny de Araújo Alves \\ Universidade Federal da Paraíba
}

\begin{abstract}
Resumo
O objetivo deste trabalho foi comparar a sensibilidade ao contraste (FSC) de crianças surdas e crianças ouvintes para freqüências espaciais de 0,25 a $2,0 \mathrm{cpg}$ (ciclos por grau de ângulo visual) em nível de luminância mesópica $\left(0,7 \mathrm{~cd} / \mathrm{m}^{2}\right)$, utilizando o método psicofísico da escolha forçada. Vinte crianças (7-12 anos) participaram desta pesquisa, dez com surdez pré-lingual e dez ouvintes. Todos os participantes apresentavam acuidade visual normal ou corrigida. A ANOVA mostrou diferença significante entre os grupos $\left[F_{(1,238)=} 15,487\right.$; $p<0,001$, porém a análise com o teste post-hoc Tukey HSD não revelou diferença significante na comparação freqüência a frequiência entre os dois grupos $(p>0,05)$. Os resultados sugerem alterações na FSC para estímulos de grade senoidal das crianças surdas em níveis de luminância mesópica.

Palavras-chave: Percepção visual; Sensibilidade ao contraste; Frequiências espaciais; Crianças surdas; Método psicofísico.
\end{abstract}

\begin{abstract}
The aim of this study was to compare the contrast sensitivity (CSF) of deaf children and hearing children to spatial frequency of 0.25 to $2.0 \mathrm{cpd}$ (cycles per degree of visual angle) at mesopic luminance level $(0.7 \mathrm{~cd} /$ $\mathrm{m}^{2}$ ), using the psychophysical forced-choice method. Twenty children (7-12 years old) participated in this research, ten with prelingual deafness and ten with normal hearing. All participants had normal or corrected visual acuity. The ANOVA showed significant difference between the two groups $\left[F_{(1,238)}=15.487 ; p<0.001\right]$, but the post-hoc Tukey HSD test analysis did not reveal significant difference in frequency to frequency comparison between the two groups $(p>0.05)$. The results suggest alterations in CSF to sine-wave gratings stimuli of deaf children at mesopic luminance level.

Keywords: Visual perception; Contrast sensitivity; Spatial frequency; Deaf children; Psychophysical method.
\end{abstract}

Uma das teorias mais utilizadas nas últimas décadas para tentar explicar como o sistema visual humano (SVH) processa a forma dos objetos foi proposta originalmente por Campbell e Robson (1968). Esta teoria, que foi denominada de modelo de canais múltiplos, defende que o processamento visual acontece através de canais que respondem seletivamente para faixas ou bandas estreitas de frequiências espaciais. A idéia é que um estímulo apresentado no campo visual não estimula todos os neurônios corticais que recebem informações daquela área da retina, mas apenas grupos de neurônios que respondem independente e seletivamente para cada um dos elementos que compõem o estímulo. Isto implica que padrões visuais diferentes são processados por subunidades ou grupos de neurônios diferentes, os quais formam as bases dos canais ou mecanis-

*Endereço para correspondência: Universidade Federal da Paraíba, Centro de Ciências Humanas Letras e Artes, Departamento de Psicologia, Laboratório de Processamento Visual Humano, Campus I, Cidade Universitária, João Pessoa, PB, Brasil, CEP 58051-900. Tel.: (83) 3216 7006; Fax: (83) 3216 7337.E-mail: natanael_labv@yahoo.com.br mos seletivos para freqüências espaciais (Campbell \& Robson, 1968; De Valois \& De Valois, 1990).

O modelo de canais múltiplos pressupõe que a função de sensibilidade ao contraste (FSC) representa o envelope de sensibilidade para a série total de canais, cada um sensível a uma faixa restrita e discreta do espectro de frequiência espacial. A FSC é a recíproca da quantidade mínima de contraste necessária para detectar um objeto qualquer ou uma grade senoidal de uma determinada freqüência espacial (Cornsweet, 1970). Assim, na medição da sensibilidade ou limiar de contraste, procura-se estabelecer experimentalmente o valor mínimo de contraste (variável dependente) que o sistema visual precisa para detectar um determinado estímulo ou freqüência espacial (variável independente). Frequiência espacial é o número de ciclos por unidade de espaço convencionalmente denominada de ciclos por grau de ângulo visual, cpg (Schwartz, 2004). Logo, uma freqüência espacial de 2,0 cpg tem duas listras claras e duas escuras em um grau de ângulo visual. O contraste é a relação entre a diferença da luminância máxima e mínima dividida pela soma da luminância máxima e mínima. 
A FSC é um indicador clássico da percepção visual humana e um dos principais instrumentos utilizados na análise teórica e clínica de estruturas da visão (Adams \& Courage, 2002). A mesma tem sido usada para avaliar e diagnosticar alterações nas vias visuais relacionadas a várias doenças, como por exemplo, estrabismo (Kiper \& Kiorpes, 1994), depressão (Cavalcanti \& Santos, 2005), intoxicação por mercúrio (Ventura et al., 2005), esquizofrenia (Slaghuis, 1998), dentre outras. Contudo, poucos pesquisadores têm utilizado a FSC para avaliar a plasticidade compensatória (compensatory plasticity) visual decorrente da surdez. Foi encontrado apenas um estudo (Finney \& Dobkins, 2001), que mediu a função de sensibilidade ao contraste de adultos surdos e ouvintes. Porém, os resultados não indicaram alterações na sensibilidade ao contraste como consequiência da surdez.

A plasticidade compensatória pode ser definida como a capacidade do cérebro de se reorganizar, de se expandir e enviar "inputs" para áreas que antes pertenciam ao sistema sensorial privado (Rauschecker, 1995). Estudos comportamentais, eletrofisiológicos e com neuroimagem realizados com pessoas cegas (Fieger, Roder, Teder-Salejarvi, Hillyard, \& Neville, 2006; Niemeyer \& Starlinger, 1981; Roder, Rosler, \& Neville, 2000) e surdas (Bavelier \& Neville, 2002; Finney, Fine, \& Dobkins, 2001; Neville \& Bavelier, 1999; Neville \& Lawson, 1987; Neville, Schmidt, \& Kutas, 1983) têm discutido a plasticidade compensatória. Por exemplo, Roder et al. (2000) utilizaram a técnica de potencial evocado auditivo para estudar o processamento de palavras em voluntários cegos. Os autores relataram que os cegos processavam palavras de maneira mais rápida do que as pessoas com visão normal.

Os trabalhos que investigam a plasticidade compensatória da percepção visual relacionada à surdez são contraditórios. Pesquisas, comparando o desempenho de participantes ouvintes e surdos em tarefas visuais cognitivas, relatam um aumento da atenção no campo visual periférico relacionado à privação auditiva (Bavelier, Dye, \& Hauser, 2006; Bavelier \& Neville, 2002; Bavelier et al., 2001; Bavelier et al., 2000; Bosworth \& Dobkins, 2002; Neville \& Lawson, 1987; Proksch \& Bavelier, 2002). Enquanto outras, relacionando busca visual e atenção, mostram que os participantes surdos apresentam prejuízos no processamento visual comparados aos ouvintes (Erden, Otman, \& Tunay, 2004; Stivalet, Moreno, Richard, Barraud, \& Raphel, 1998). Já trabalhos que compararam o desempenho sensorial de crianças e adultos surdos e ouvintes, utilizando o paradigma da detecção de sinais (Bross, 1979a, 1979b; Bross \& Sauerwein, 1980), não encontraram alterações na resposta sensorial entre os participantes com e sem surdez. Em termos gerais, há pelo menos duas hipóteses: a primeira defende que apenas alguns aspectos da visão são modificados em função da surdez (Bavelier et al., 2006) e a segunda defende que as contradições entre os estudos estão relacionadas às diferentes metodologias e critérios de amostragem das pesquisas (Bavelier et al., 2006; Rettenbach, Diller, \& Sireteanu, 1999; Stevens \& Neville, 2006).
O presente estudo determinou a FSC de crianças surdas e ouvintes para grades senoidais verticais, estáticas e em condições baixas ou mesópicas de luminância, utilizando o método psicofísico da escolha forçada. O objetivo foi verificar se a surdez altera a percepção visual ou as vias visuais que processam estímulos acromáticos de freqüência espacial baixa. A idéia fundamental em relação à utilização da FSC é que a redução ou a elevação do limiar sensório é um sinal de alterações nas vias sensórias que processam contraste decorrente da privação sensorial ou da plasticidade compensatória. Estudos relacionando a FSC e os efeitos da surdez na plasticidade compensatória visual são inexistentes em crianças e raros em adultos. Pois, a maioria dos estudos investiga aspectos cognitivos e perceptuais superiores, como por exemplo, memória e habilidades verbais.

\section{Método}

\section{Participantes}

Participaram dos experimentos 20 crianças de ambos os sexos com idades entre 7 e 12 anos, 10 com audição normal $(M=11,3 ; D P=1,79)$ e 10 com surdez $(M=11,5 ; D P$ $=1,78)$. Todas as crianças apresentavam acuidade visual normal ou corrigida e não tinham histórico de patologia ocular. A acuidade visual foi medida com a cartela de optotipos "E" de Rasquin.

As crianças com perda auditiva apresentavam surdez pré-lingual (adquirida em um período que antecede a linguagem falada) bilateral, de grau severo a profundo (perda auditiva $\geq 80 \mathrm{~dB}$ ), sendo que oito apresentavam surdez congênita (cinco decorrente de causa desconhecida e três de rubéola materna) e duas apresentavam surdez adquirida (uma decorrente de meningite e a outra do efeito de medicamentos). As crianças surdas sabiam a Língua Brasileira de Sinais (LIBRAS), foram diagnosticadas com surdez por médicos e fonoaudiólogos e encaminhadas ao laboratório pelo Sistema Universal Verbotonal da Audição Guberina (SUVAG) e pelo o Centro de Educação Permanente para Surdos (CEPES).

A participação na pesquisa ocorreu mediante a assinatura de um termo de consentimento livre e esclarecido pelos responsáveis, conforme a resolução no 196/96 do Conselho Nacional de Saúde (Ministério da Saúde), que trata das diretrizes e normas de pesquisas envolvendo seres humanos. Este estudo foi aprovado pelo Comitê de Ética em Pesquisa, do Centro de Ciências da Saúde (CEP/CCS).

Pesquisas empíricas que utilizam limiares sensórios, métodos psicofísicos e medidas repetidas, envolvem um número pequeno de voluntários em cada condição, uma vez que estão sendo avaliados aspectos automáticos ou processos sensoriais da percepção (Finney \& Dobkins, 2001; França, Santos, \& Mendes, 2006).

\section{Equipamentos e Estímulos Visuais}

Os estímulos visuais foram gerados em um monitor de vídeo CLINTON MEDICAL de 21 polegadas, digital, monocromático, de alta resolução. A luminância média da 
tela do monitor foi de $0,7 \mathrm{~cd} / \mathrm{m}^{2}$ (condições mesópicas), ajustada com um fotômetro do tipo SPOT METTER, com a precisão de um grau (ASAHI PENTAX). A definição de luminância mesópica varia muito entre os estudos encontrados na literatura, entretanto alguns autores consideram como luminância mesópica o intervalo entre 0,003 a $3 \mathrm{~cd} /$ $\mathrm{m}^{2}$ (Ketomäki, Eloholma, Orreveteläinen, \& Halonen, 2003).

Foram utilizados um estímulo neutro com a luminância homogênea e estímulos de grades senoidais verticais com frequiências espaciais de 0,$25 ; 0,5 ; 1$ e $2 \mathrm{cpg}$ (ciclos por grau de ângulo visual). Estes eram circulares com um diâmetro de aproximadamente 7 graus de ângulo visual a 150 $\mathrm{cm}$ de distância da tela (Figura 1). A escolha dos estímulos acima foi baseada na idéia geral de que as frequiências espaciais são processadas por canais interdependentes e seletivos para aproximadamente uma oitava de frequiência (Blakemore \& Campbell, 1969a, 1969b; Campbell \& Maffei, 1970). Outras informações sobre os estímulos visuais do tipo grade senoidal podem ser encontradas na literatura (Santos, Simas, \& Nogueira, 2003; Schwartz, 2004).
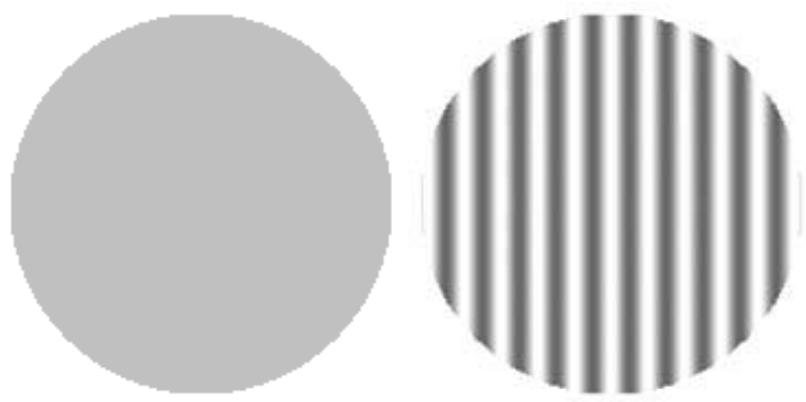

Figura 1. Exemplos de um par de estímulos, à esquerda estímulo neutro com luminância média e à direita uma grade senoidal com freqüência espacial de 1,0 cpg. Estímulos originalmente calibrados para serem vistos a $150 \mathrm{~cm}$.

\section{Procedimento}

O método psicofísico da escolha forçada (Wetherill \& Levitt, 1965), também descrito em França et al. (2006) e Santos et al. (2003), calcula a probabilidade de acertos consecutivos do observador. De acordo com este método, o voluntário tem de escolher entre dois estímulos àquele que contém a freqüência de teste (grade senoidal).

Durante cada sessão experimental, os participantes foram submetidos à discriminação sucessiva simples de pares de estímulos na tela do monitor. A ordem de apresentação aleatória dos estímulos foi controlada pelo computador. Cada estímulo foi apresentado por dois segundos, com um intervalo entre um e outro de um segundo. Os intervalos entre as tentativas (ou julgamentos do voluntário) foram em média de três segundos.

Todas as medições foram realizadas a uma distância de $150 \mathrm{~cm}$ da tela do monitor, com visão binocular e pupila natural. Antes do início de cada sessão experimental, os voluntários foram orientados a pressionar o botão esquerdo do mouse (ou número 1) quando julgassem que o estímulo de teste foi apresentado primeiro, e o botão direito do mouse (ou número 2) quando julgassem que o estímulo de teste foi apresentado em segundo lugar. Os voluntários receberam a seguinte instrução:

será apresentada uma sequiência de pares de estímulos, um com listras claras e escuras e outro totalmente cinza. Você deverá escolher sempre o estímulo que contém as listras, pressionando o botão do lado esquerdo (botão número 1) do mouse quando o estímulo com listras for apresentado primeiro e, o botão do lado direito (botão número 2), quando for apresentado em segundo lugar (após o estímulo cinza).

As sessões foram iniciadas com o contraste do estímulo teste em nível supralimiar e os experimentos só começaram quando o experimentador certificou-se que os participantes entenderam e responderam conforme as instruções. As instruções para os participantes surdos foram dadas em LIBRAS.

Cada freqüência foi estimada duas vezes, em dias diferentes. A ordem de mensuração das frequiências foi aleatória. Os experimentos foram realizados em um ambiente com pouca iluminação. O critério adotado para mensurar as curvas foi o de três acertos consecutivos para diminuir uma unidade de contraste, e o de um erro para aumentar o contraste em uma unidade $(0,08 \%)$.

O número de apresentações necessárias para a determinação do limiar de contraste variou de acordo com os acertos do participante. Cada sessão foi finalizada automaticamente pelo programa quando três valores máximos e três valores mínimos de contraste foram obtidos. No total, foram medidas 40 curvas de sensibilidade ao contraste, 20 curvas para as crianças surdas e 20 para as crianças ouvintes com audição normal.

Após cada sessão, o programa produziu uma folha de resultados com a situação experimental e os seis valores de contraste conseguidos pelas reversões. Os valores de contraste obtidos para cada freqüência foram agrupados em planilhas por condição (surdez e ouvinte) e a grande média foi utilizada como estimativa da sensibilidade ao contraste em função da freqüência espacial.

\section{Resultados}

A Figura 2 mostra as curvas de FSC de crianças surdas e de crianças ouvintes para grades senoidais de freqüência espacial de 0,$25 ; 0,5 ; 1,0$ e 2,0 cpg. A sensibilidade ao contraste (FSC) é o inverso do limiar de contraste (1/FSC). Deste modo, quanto menor o limiar de contraste maior a sensibilidade do sistema visual e vice-versa. As barras verticais em cada uma das curvas de FSC indicam os erros padrões das médias.

Os resultados mostraram que a máxima sensibilidade ocorreu na faixa de freqüência de $0,5 \mathrm{cpg}$ para as crianças surdas e na frequiência $0,5 \mathrm{cpg}$ para as crianças ouvintes. Enquanto a menor sensibilidade ocorreu na freqüência de 2,0 cpg para os dois grupos. Os resultados mostram ainda 


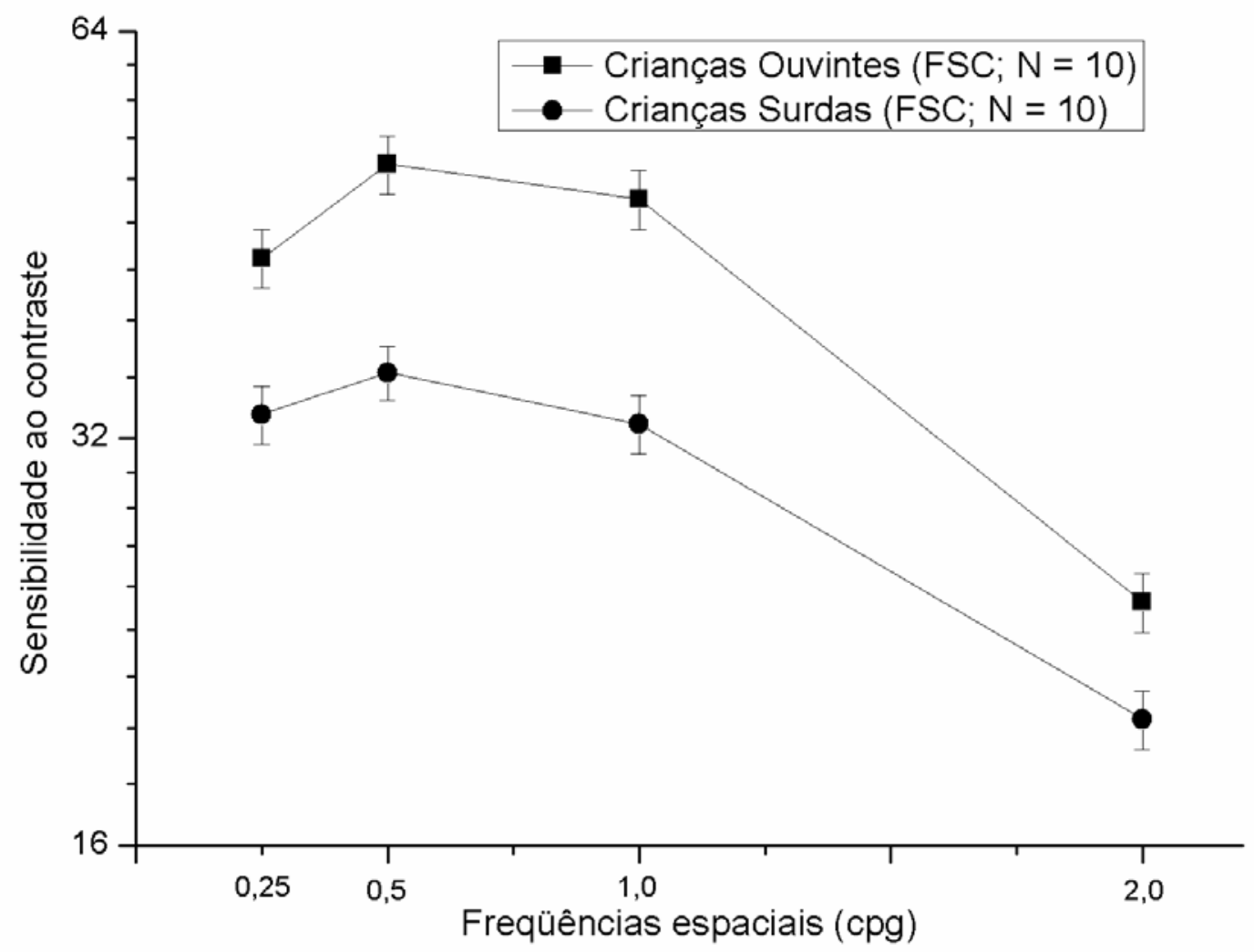

Figura 2. Curvas de sensibilidade ao contraste para freqüências espaciais em crianças surdas ( ——) e crianças com audição normal ( $\longrightarrow$ ), com idades entre 7 e 12 anos. As linhas verticais mostram o erro padrão da média para cada freqüência $(0,25 ; 0,5 ; 1,0$ e 2,0 cpg).

que as crianças surdas precisaram da ordem de 1,$3 ; 1,5$; 1,4 e 1,2 mais contraste do que as crianças ouvintes para perceberem as freqüências espaciais de 0,$25 ; 0,5 ; 1,0$ e 2,0 cpg, respectivamente.

A ANOVA simples revelou diferenças significantes entre a FSC dos dois grupos $\left[F_{(1,238)}=15,487 ; p<0,001\right]$. A análise com o teste post-hoc Tukey HSD não revelou diferença significante na comparação freqüência a frequiência entre os dois grupos $(p>0,05)$. Estes resultados mostram diferenças significantes entre as médias gerais dos dois grupos, indicando que as crianças ouvintes podem ser mais sensíveis do que as crianças surdas para grades senoidais com freqüências espaciais em condições mesópicas.

\section{Discussão}

O presente estudo teve como objetivo determinar o limiar sensório (FSC) visual de crianças surdas e de crianças ouvintes para frequiências espaciais com método psicofísico da escolha forçada e verificar se a surdez altera a percepção visual de contraste ou as vias visuais que processam estímulos acromáticos de baixa frequiência em níveis baixos de luminância.

A comparação entre as curvas de FSC de crianças surdas e de crianças ouvintes de 7 a 12 anos mostrou que a FSC dos dois grupos apresentam formas semelhantes. Isto é, cada curva apresenta uma faixa de maior sensibilidade em torno de $0,5 \mathrm{cpg}$; diminuindo para a esquerda $(0,25 \mathrm{cpg}) \mathrm{e}$ para a direita (1,0 e 2,0 cpg). Observa-se que o sistema é menos sensível à medida que se afasta da banda de máxima sensibilidade (Figura 2).

Os resultados mostraram diferenças estatísticas entre a sensibilidade ao contraste média das crianças surdas e das crianças ouvintes para freqüências espaciais em condições mesópicas $\left[F_{(1,238)}=15,487 ; p<0,001\right]$, demonstrando que as crianças ouvintes apresentaram limiares de contraste médios de aproximadamente 1,4 vezes menores que as crianças surdas. Entretanto, o teste post-hoc Tukey não revelou diferença significante entre as freqüências $(p>0,05)$. Isto significa que embora o padrão de comportamento da FSC indique diferença entre os dois grupos (ver Figura 2), os resultados não são conclusivos, pois o teste post-hoc não revelou diferenças entre os dois grupos em nenhuma das freqüências testadas.

O fato do teste post-hoc não ter demonstrado diferença significante pode estar relacionado a fatores cognitivos como critério de escolha de resposta, atenção e motivação dos participantes que são atributos críticos em pesquisas sobre limiar sensório. Entretanto, o método da escolha forçada utilizado no presente estudo é um paradigma bem estabelecido que minimiza os efeitos de critério de escolha de resposta dos participantes (Schwartz, 2004). Inclusive, o contraste só era reduzido em uma unidade $(0,08 \%)$ após três acertos consecutivos, o que excluía a resposta ao acaso, uma vez que seria muito difícil para o participante acertar três vezes consecutivas sem realmente perceber o 
estímulo. Entretanto, o método psicofísico da escolha forçada pode sofrer influências da atenção e da vontade do participante, pois ele tem que querer acertar. A atenção e a motivação podem ser problemas para as crianças, mas os experimentos foram conduzidos de forma criteriosa, de modo que diante de qualquer sinal de falta de interesse ou de cansaço (por exemplo, bocejar e olhar para os lados) os experimentos foram encerrados. Além disto, neste tipo de estudo, trabalha-se com a hipótese de que o voluntário tem de acertar sempre, quando a criança erra com frequiência pode ser um sinal de falta de motivação e atenção. De qualquer forma, é cedo para afirma que a plasticidade compensatória, procedente da privação auditiva, altera a FSC das crianças surdas quando comparadas às crianças ouvintes.

Por outro lado, qualquer comparação entre este estudo e outros trabalhos encontrados na literatura é inviável, independente de corroborar ou não a hipótese que a plasticidade compensatória decorrente da privação auditiva altera a percepção ou processamento visual. Isto porque os estudos foram realizados com pressupostos teóricos e metodológicos tão diferentes que inviabilizam qualquer comparação. Os mesmos vão desde aqueles que não encontraram alterações sensoriais com o paradigma da detecção de sinais (Bross, 1979a, 1979b; Bross \& Sauerwein, 1980) e a FSC em adultos (Finney \& Dobkins, 2001), até aqueles que encontraram melhoras (Bavelier \& Neville, 2002; Bosworth \& Dobkins, 1999, 2002; Neville \& Lawson, 1987; Proksch \& Bavelier, 2002; Sladen, Tharpe, Ashmead, Grantham, \& Chun, 2005) ou prejuízos em tarefas envolvendo atenção visual (Erden et al., 2004; Stivalet et al., 1998).

Por fim, a maior parte dos estudos na literatura utiliza condições fotópicas, ou seja, níveis altos de luminância (Finney \& Dobkins, 2001; Neville \& Lawson, 1987; Proksch $\&$ Bavelier, 2002; Sladen et al., 2005), enquanto o presente estudo utilizou níveis baixos de luminância (condições mesópicas). É importante ressaltar que existem pelo menos duas vias paralelas com estruturas e funções diferentes envolvidas no processamento visual de objetos e contraste: a via visual parvocelular $(\mathrm{P})$, que é especializada no processamento de freqüências espaciais médias e altas ou detalhes finos e opera em níveis altos luminância, e a via visual magnocelular (M), que é especializada no processamento de freqüências espaciais baixas e opera em níveis baixos de luminância (Benedek, Benedek, Kéri, \& Janáky, 2003; Neville \& Bavelier, 1999). Nesta direção, pode ser que a surdez se correlacione de maneira diferente com o sistema nervoso e as vias P e M. Esta hipótese e os achados do presente estudo precisam ser melhor investigados.

\section{Considerações Finais}

É cedo para afirmar que a plasticidade compensatória altera a FSC ou a percepção visual de contraste. Os resultados apresentados neste estudo são iniciais e novas pesquisas precisam ser realizadas para aprofundar e produzir novos dados empíricos com estímulos e níveis de luminância diferentes. Só assim será possível discutir de maneira mais sistemática as possíveis alterações na percepção visual relacionadas à surdez.

\section{Referências}

Adams, R. J., \& Courage, M. L. (2002). Using a single test to measure human contrast sensitivity from early childhood to maturity. Vision Research, 42, 1205-1210.

Bavelier, D., Brozinsky, C., Tomann, A., Mitchell, T., Neville, H., \& Liu, G. (2001). Impact of early deafness and early exposure to sign language on the cerebral organization for motion processing. The Journal of Neuroscience, 21(22), 89318942.

Bavelier, D., Dye, M. W. G., \& Hauser, P. C. (2006). Do deaf individuals see better? Trends in Cognitive Sciences, 10(9), 391-430.

Bavelier, D., \& Neville, H. J. (2002). Cross-modal plasticity: Where and how? Neuroscience, 3, 443-452.

Bavelier, D., Tomann, A., Hutton, C., Mitchell, T., Corina, D., Liu, G., et al. (2000). Visual attention to the periphery is enhanced in congenitally deaf individuals. The Journal of Neuroscience, 20(17), 1-6.

Benedek, G., Benedek, K., Kéri, S., \& Janáky, M. (2003). The scotopic low-frequency spatial contrast sensitivity develops in children between the ages of 5 and 14 years. Neuroscience Letters, 345, 161-164.

Blakemore, C., \& Campbell, F. C. (1969a). Adaptation to spatial stimuli. Journal of Physiology, 200, 11-13P.

Blakemore, C., \& Campbell, F. C. (1969b). On the existence of neurons in the human visual system selectively sensitive to the orientation and size of retinal images. Journal of Physiology, 203, 237-260.

Bosworth, R. G., \& Dobkins, K. R. (1999). Left-hemisphere dominance for motion processing in deaf signers. Psychological Science, 10(3), 256-262.

Bosworth, R. G., \& Dobkins, K. R. (2002). The effects of spatial attention on motion processing in deaf signers, hearing signers and hearing nonsigners. Brain and Cognition, 49(1), 152-169.

Bross, M. (1979a). Residual sensory capacities of the deaf: A signal detection analysis of a visual discrimination task. Perceptual and Motor Skills, 48, 187-194.

Bross, M. (1979b). Response bias in deaf and hearing subjects as a function of motivational factors. Perceptual and Motor Skills, 49, 779-782.

Bross, M., \& Sauerwein, H. (1980). Signal detection analysis of visual flicker in deaf and hearing individuals. Perceptual and Motor Skills, 51, 839-843.

Campbell, F. W., \& Maffei, L. (1970). Electrophysiologycal evidence for the existence of orientation and size detectors in the human visual system. Journal of Physiology, 207, 635-652.

Campbell, E. W., \& Robson, F. G. (1968). Application of the Fourier analysis to the visibility of gratings. Journal of Physiology, 197, 551-566.

Cavalcanti, A. M., \& Santos, N. A. (2005). O efeito da depressão na função de sensibilidade ao contraste em humanos: Achados preliminares. Revista Brasileira de Terapias Cognitivas, 1(1), 21-27.

Cornsweet, T. N. (1970). Vision perception. New York: Academy Press.

De Valois, R. L., \& De Valois, K. K. (1990). Spatial vision (2nd ed.). New York: Oxford University Press.

Erden, Z., Otman, S., \& Tunay, V. B. (2004). Is visual perception of hearing-impaired children different from healthy children? International Journal of Pediatric Otorhinolaryngology, 68, 281-285. 
Fieger, A., Roder, B., Teder-Salejarvi, W., Hillyard, S. A., \& Neville, H. J. (2006). Auditory spatial tuning in late-onset blindness in humans. Journal of Cognitive Neuroscience, 18(2), 149-157.

Finney, E. M., \& Dobkins, K. R. (2001). Visual contrast sensitivity in deaf versus hearing populations: Exploring the perceptual consequences of auditory deprivation and experience with a visual language. Cognitive Brain Research, 11(1), 171-183.

Finney, E. M., Fine, I., \& Dobkins, K. R. (2001). Visual stimuli activate auditory cortex in the deaf. Nature Neuroscience, 4(12), 1171-1173.

França, V. C. R. M., Santos, N. A., \& Mendes, L. C. (2006). Sensibilidade ao contraste em crianças pré-escolares com método psicofísico. Psicologia: Teoria e Pesquisa, 22(3), 335338.

Ketomäki, J., Eloholma, M., Orreveteläinen, P., \& Halonen, L. (2003). Mesopic lighting conditions and pedestrian visibility. Ingineria Iluminatului, 11, 29-35.

Kiper, D. C., \& Kiorpes, L. (1994). Suprathreshold contrast sensitivity in experimentally strabismic monkeys. Vision Research, 34(12), 1575-1583.

Neville, H. J., \& Bavelier, D. (1999). Specificity and plasticity in neurocognitive development in humans. In M. Gazzaniga (Ed.), The new cognitive neurosciences (2nd ed., pp. 83-98). Cambridge, MA: MIT Press.

Neville, H. J., \& Lawson, D. (1987). Attention to central and peripheral visual space in a movement detection task. III. Separate effects of auditory deprivation and acquisition of a visual language. Brain Research, 405(2), 284-294.

Neville, H. J., Schmidt, A., \& Kutas, M. (1983). Altered visualevoked potentials in congenitally deaf adults. Brain Research, 266(1), 127-132.

Niemeyer, W., \& Starlinger, I. (1981). Do the blind hear better? Investigations on auditory processing in congenital or early acquired blindness. II. Central functions. Audiology, 20(6), 510-515.

Proksch, J., \& Bavelier, D. (2002). Changes in the spatial distribution of visual attention after early deafness. Journal of Cognitive Neuroscience, 14(5), 687-701.

Rauschecker, J. P. (1995). Compensatory plasticity and sensory substitution in the cerebral córtex. Trends in Neurosciences, 18(1), 36-43.

Rettenbach, R., Diller, G., \& Sireteanu, R. (1999). Do deaf people see better? Texture segmentation and visual search compensate in adult but not in juvenile subjects. Journal of Cognitive Neuroscience, 11(5), 560-575.

Roder, B., Rosler, F., \& Neville, H. J. (2000). Event-related potentials during auditory language processing in congenitally blind and sighted people. Neuropsychologia, 38(11), 14821502.

Santos, N. A., Simas, M. L. B., \& Nogueira, R. M. T. B. L. (2003). Processamento visual da forma em idosos: Curvas de limiar de contraste para freqüências angulares e senoidais. Psicologia: Reflexão \& Crítica, 16(2), 271-277.

Schwartz, S. H. (2004). Visual perception: A clinical orientation (3nd ed.). New York: McGraw Hill.

Sladen, D. P., Tharpe, A. M., Ashmead, D. H., Grantham, D. W., \& Chun, M. M. (2005). Visual attention in deaf and normal hearing adults: Effects of stimulus compatibility. Journal of Speech, Language, and Hearing Research, 48, 1529-1537.

Slaghuis, W. L. (1998). Contrast sensitivity for stationary and drifting spatial frequency gratings in positive and negativesymptom schizophrenia. Journal of Abnormal Psychology, 107(1), 49-62.
Stevens, C., \& Neville, H. (2006). Neuroplasticity as a doubleedged sword: Deaf enhancements and dyslexic deficits in motion processing. Journal of Cognitive Neuroscience, 18(5), 701-714.

Stivalet, P., Moreno, Y., Richard, J., Barraud, P., \& Raphel, C. (1998). Differences in visual search tasks between congenitally deaf and normally hearing adults. Cognitive Brain Research, 6(3), 227-232.

Ventura, D. F, Simões, A. L., Tomaz, S., Costa, M. F., Lago, M., Costa, M. T. V., et al. (2005). Colour vision and contrast sensitivity losses of mercury intoxicated industry in Brazil. Environmental Toxicology and Pharmacology, 19, 523-529.

Wetherill, G. B., \& Levitt, H. (1965). Sequential estimation of points on a psychometric function. The British Journal of Mathematical and Statistical Psychology, 48, 1-10.
Recebido: 05/12/2007 $1^{a}$ revisão: $15 / 07 / 2008$ $2^{a}$ revisão: 17/09/2008 Aceite final: 17/10/2008 